\title{
Research on After-sales Quality Problem Management of Electrical Connectors under Aerospace Product R\&D System
}

\author{
Hui Zhang ${ }^{1, a}$, Meiqing Wang ${ }^{2, b}$ \\ ${ }^{1}$ School of Mechanical Engineering and Automation, Beihang University, Beijing, China, 100191 \\ ${ }^{2}$ School of Mechanical Engineering and Automation, Beihang University, Beijing, China, 100191 \\ afty121305@163.com, bwangmq@buaa.edu.cn
}

\begin{abstract}
Keywords: Aerospace product R\&D system; Electrical connectors; Product after-sales quality problems processing; Information systems;

Abstract. In order to improve the processing efficiency of after-sales quality problem for aerospace electrical connector, the approach of after-sales quality problem management is studied. The requirements of after-sales quality problem management under the information environment are analyzed. The process model of after-sales quality problem management is setup, in which the multi-types of quality problem handling method are considered. The multi-layer software system architecture for after-sales quality problem management is provided. The information management system of after-sales quality problem is developed by using J2EE architecture, and the feasibility and validity of the proposed method are verified by applied the software system in an aerospace electrical connector manufacturer.
\end{abstract}

\section{Introduction}

In electronic and control systems, electrical connectors are the basic components which are necessary to form a complete system. Its main function is electrical connection and signal transmission between devices and components, components and components, systems and systems [1]. In all types of weapons and equipment, electrical connectors have been used frequently. A complex space system usually has thousands of various types of electrical connectors, such as a certain type of launch task which requires more than 3,400 sets of various electrical connectors just for the ground equipment. [2] Therefore, as one of the aerospace electronic components, the electrical connector plays an important part in aerospace products.

China's aerospace products is known to the world for its high reliability, high emission success rate. The success is based on the strict close loop management system of quality problems for the aerospace products. The guideline of aerospace quality problem management, which is called "double-close loop", has become industry standards in Chinese space industry quality management after its development for more than 20 years. Supporting enterprises for aerospace product are supposed to not only implement the quality management according to the internal quality assurance outline, but also carry out quality control and management activities with users for their product quality management needs. This requires enterprises to clarify the mechanism of the problem through the analysis of the problem, to put forward effective measures to improve, and to avoid similar problems occurring in other types of products by analogy.

As a bridge between enterprises and users, after-sales quality management department not only acts as the receiver of the quality of after-sales problems, but also deals with the quality problems and monitors the whole implementation process. Due to the characters of aerospace products: the various product's components, the difficult $\mathrm{R} \& \mathrm{D}$ technology, the complicated manufacturing process and the multi-sectoral collaborated project management, the complexity of the quality problems of aerospace products is different, so the complexity of the process varies.

Simple after-sales quality problems can be handled in field or by returning the problem product to factory for repairs, and serious quality problems need to be closed loop. Besides, with the researchers carrying out the in-depth analysis on the mechanism of the quality problems, they may discover serious problems and consequences hidden behind the original seemingly simple problems. As a result, the 
seriousness of the quality problem will be upgraded, the corresponding problem-treatment level will be improved.

Therefore, there are many features in after-sales quality problem treatment: various types of problem and implement flow, complex problem processing flow, a wide variety of problem handling states, etc. This has brought great challenges to the after-sales quality control personnel. The original manual processing method is only suitable for the simple management of the problem, it is difficult to realize the reuse of the valuable information, the tracking of the complex process, and the efficient collaboration in handling after-sales quality problem. Hence, there is an urgent need to enhance the efficiency of the after-sales quality problem management efficiency and the degree of refinement by information technology.

\section{Demand Analysis of After-sales Quality Problem Management}

Quality Problem Handling Mechanism in Aerospace Product R\&D. The electrical connectors are the important ancillary products for aerospace products. Its quality problem management work also needs to meet the general quality management practices for components. For an aerospace product, its supporting components are generally under such aspects of quality control, like the quality control in the process of selection, procurement, production supervision, inspection, arrival inspection, supplementary screening, assembly and commissioning, etc. The solution to component problems which appear on different links, requires carrying out failure analysis to find out the hidden reasons [3]. At the same time, there is a need to establish closed-loop control in component quality management, to improve the component quality and reliability and to ensure that the component meets the needs of aerospace products. [4]

Components product quality assurance process for the aerospace product $R \& D$ system involves different operations like selection, procurement, supervision and acceptance, screening, re-inspection, installed control, etc. If the whole machine failure occurred when the whole machine was running, the failure analysis should be carried out step by step, tracing back from Aerospace General Academy to sub-system plants or institutes, in order to find out the mechanism of the failure or the failure component. The detailed process is shown in Fig.1.

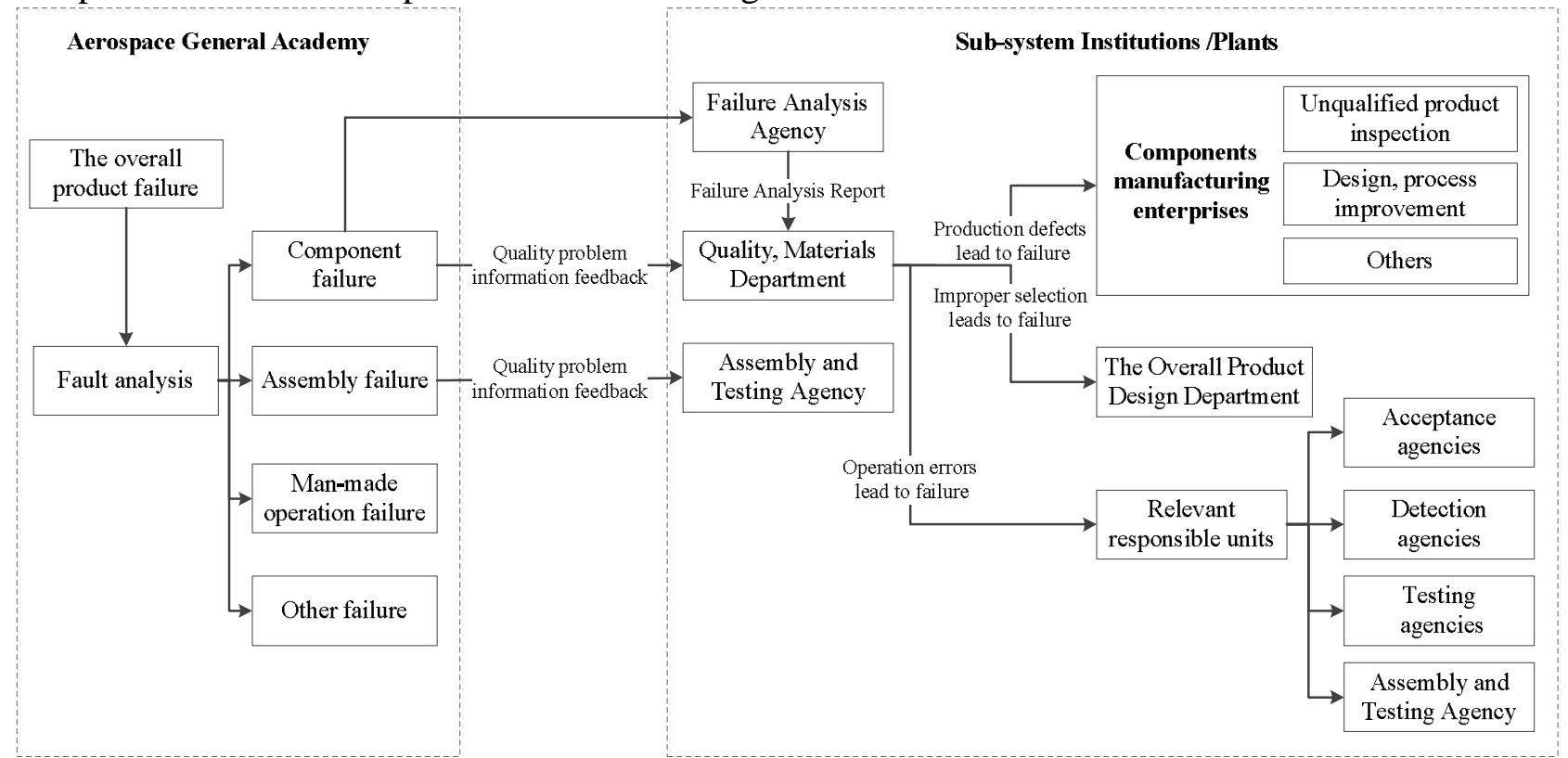

Fig.1 A flow chart for quality traceability of component failure under aerospace product R\&D system

After-sales Quality Management Process. Generally, the traditional product after-sales service process is this: first, the enterprise receives the customer feedback information (possibly by the incoming call or e-mail, the incoming person and so on) and then it carries on the classification based on the complexity degree of the problem. 
For simple quality problems, the company sent a service staff to the spot to maintain, or returned the failure product back to the factory for factory processing. For complex quality problems, enterprises will, in accordance with the needs of users, deal with the problem by methods like problem correction, quality problem close loop, etc. If the quality problem had been found to have a larger impact to other products during the handling process, the problem information should be transmitted to the relevant institutes and plants, in order to check whether there is a similar problem in their enterprises and take preventive measures as soon as possible.[5]

Consequently, through the research on the after-sales quality management mode of electrical connector manufacturing enterprises, this paper summarizes the following aspects of the improvement needs in the traditional after-sales quality management mode:

ü The accumulation of quality problem-solving knowledge: in the existing problem-solving cases of electrical connectors manufacturing enterprises, most of them are being treated separately, besides, the information of problems haven't been sorted out and transformed into knowledge for storage, so that the utilization of historical information is low and it is a waste of resources of information in a large degree;

ü The improvement of the speed and accuracy of after-sales response: the poor service response results in bad service. For some products, the enterprises cannot respond to the customers' requests in time because of the limitation of time, place and resources. In addition, in order to identify the user needs accurately, focus on the mechanism of the quality problem quickly, match the problem with effective solution and make appropriate response to the users to enhance customer satisfaction, the enterprises should make a further improvement in the enterprise management;

ü Life-cycle management of problem-solving: when enterprises deal with their after-sales quality problems, they focus more on whether the single link of the issue is completed or not, and not on the effective management for the whole problem-solving process. It is easy to result in the low efficiency of the problem-solving and the poor reception of information for the relevant person who is in charge of handling the whole process of dealing with quality problems;

ü Cooperation of quality problem-solving: for complex after-sales quality problems, they often need multi-sector cooperation during the problem-solving process. In cross-sectoral information transmission of the enterprise, problems like incomplete information, loss of information transmission and other problems often occur. So an information-sharing platform for different departments, different posts is necessary, it enables people to receive information timely and enhances the efficiency of collaborative problem-solving;

ü Traceability of quality problem: for the problem which should be closed loop, the key problem information should be provided for relevant institutes and plants, so that they can carry out the recheck of various types of products, the inspection of enterprise management norms, the review of various types of design process documents, to ensure the quality problem can be solved and prevented systematically and be closed loop in time in order to avoid unnecessary economic losses;

ü Improvement of quality management system: After the quality problems are solved, a lot of quality information is simply stored in the database as archive data, and the accumulated information is not analyzed effectively. The multidimensional, multi-level statistical analysis can help enterprises find bottlenecks in quality management and support the improvement of enterprise quality management system, thus reducing the occurrence of after-sales quality problems and improving the efficiency of quality problems solving.

Hence, enterprises need a set of information management software for unified after-sales quality problem management, to achieve the rapid response of field feedback quality problems, the automated, standardized problem-tracking and the closed-loop quality problem management. At the same time, the typical quality problem-solving methods are sorted out in the form of cases for storage and standardized as the failure modes, so as to accumulate the knowledge of enterprise quality problems and improve the quality problem-solving capacity; In addition, the use of information technology can 
achieve the data statistics and management analysis of quality problems, and promote the continuous improvement of process management for quality problem-solving.

\section{After-sales Quality Problem Management Process Modelling}

As a contractor of aerospace product component, electrical connector manufacturing enterprises need to work with relevant departments and institutes to solve different quality problems collaboratively based on the whole $R \& D$ process of aerospace product.

The process diagram for after-sales quality problem management of electrical connector enterprise are shown in Fig.2:

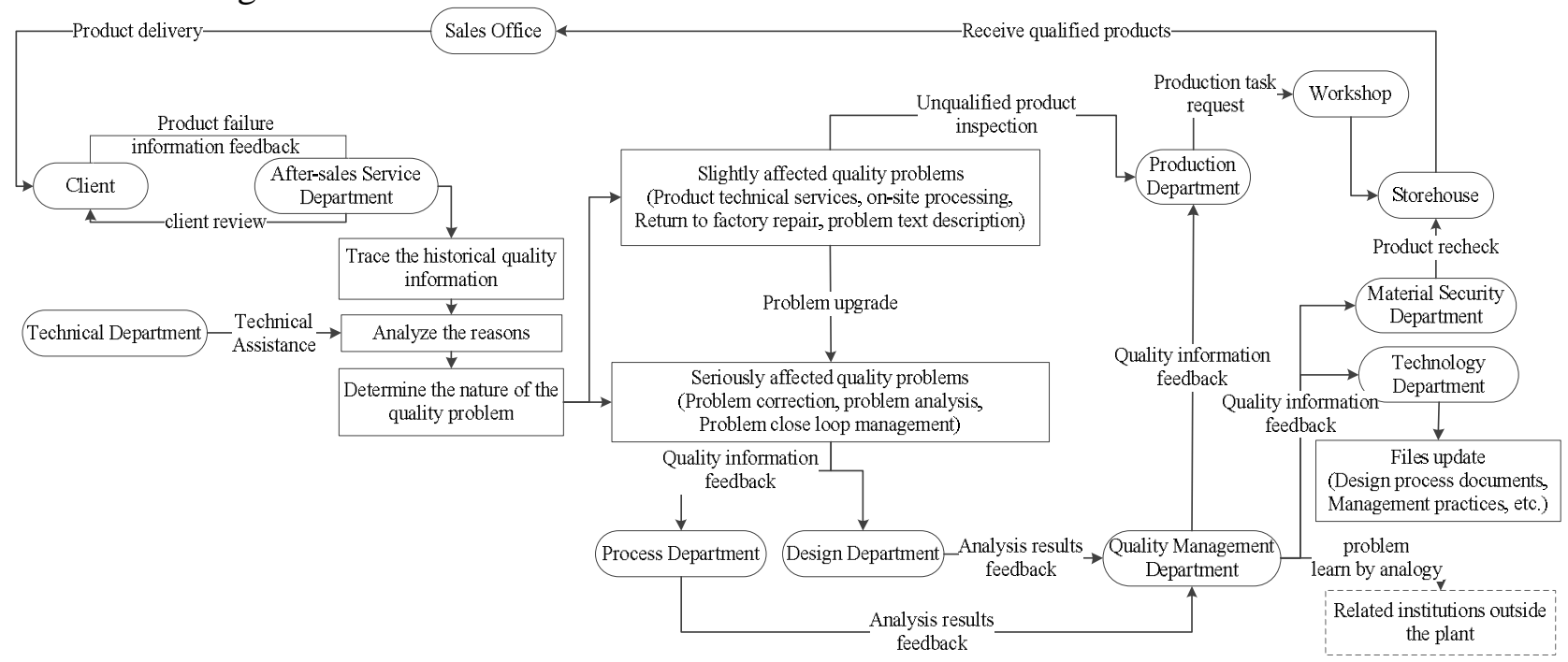

Fig.2 A flow chart for after-sales quality problem management in enterprise

When the failure product information from the customer is received, the after-sales service department will first analyze the reason for the product failure taking the product's historical quality information as reference and they sometimes need technically support in the process. Secondly, the after-sales service department can determine the problem-solving method after identifying the nature of the problem.

For seriously affected quality problems, they need to enter the seriously affected quality problems solving process. In accordance with the "five-close-loop" requirements, the process will carry out close loop issues in various departments and institutes, like design department, process department, quality department, science and technology department, material security department, production department, workshop, warehouse, or even outside institutes like the relevant units and institutions under similar system; for the relatively small quality problems, they will enter the smaller quality problems solving process. The after-sales service department will provide customers after-sale services like providing technical advice for customers, on-site service and so on. For the failure products which should be returned to factory for further repair, the after-sales service department should timely submit the substandard product disposal request to production office, the production office will assign the production tasks to workshop for the failure product to rework or repair. If the staffs find that the identification of the problem nature is not accurate, or the choice of problem treatment is not appropriate, they can upgrade the treatment of quality problem or transform it into other methods. This can ensure that all quality problems to be handled can be properly addressed.

After modeling and analyzing the after-sales quality problem management process, we can find that to tackle with any kind of after-sales quality problem, the faulty product field information should be collected. Besides, when tracing the historical quality information or judging the nature of the quality problem, if the multi-dimensional quality information of faulty product or the previous problem-solving information can be effectively reused, it will do a good favor to judge the quality of the problem and improve the efficiency of problem-processing greatly. In addition, monitoring the whole process for 
different solutions will help to determine the nature of the problem during the process assessment and upgrade the problem treatment in time according to the problem in-depth development.

\section{After-sales Quality Management System Design}

Software System Architecture. Based on the above analysis, this paper constructs an after-sales quality problem management information system for electrical connectors manufacturing enterprises under aerospace product $\mathrm{R} \& \mathrm{D}$ system. The system is aimed to provide enterprises with a management plan which is based on the feedback of field quality problems, supported by the quality problem case base and failure mode library, and it will achieve the monitoring of the whole process of quality problems handling. The multi-layer software system architecture is shown in Fig.3.

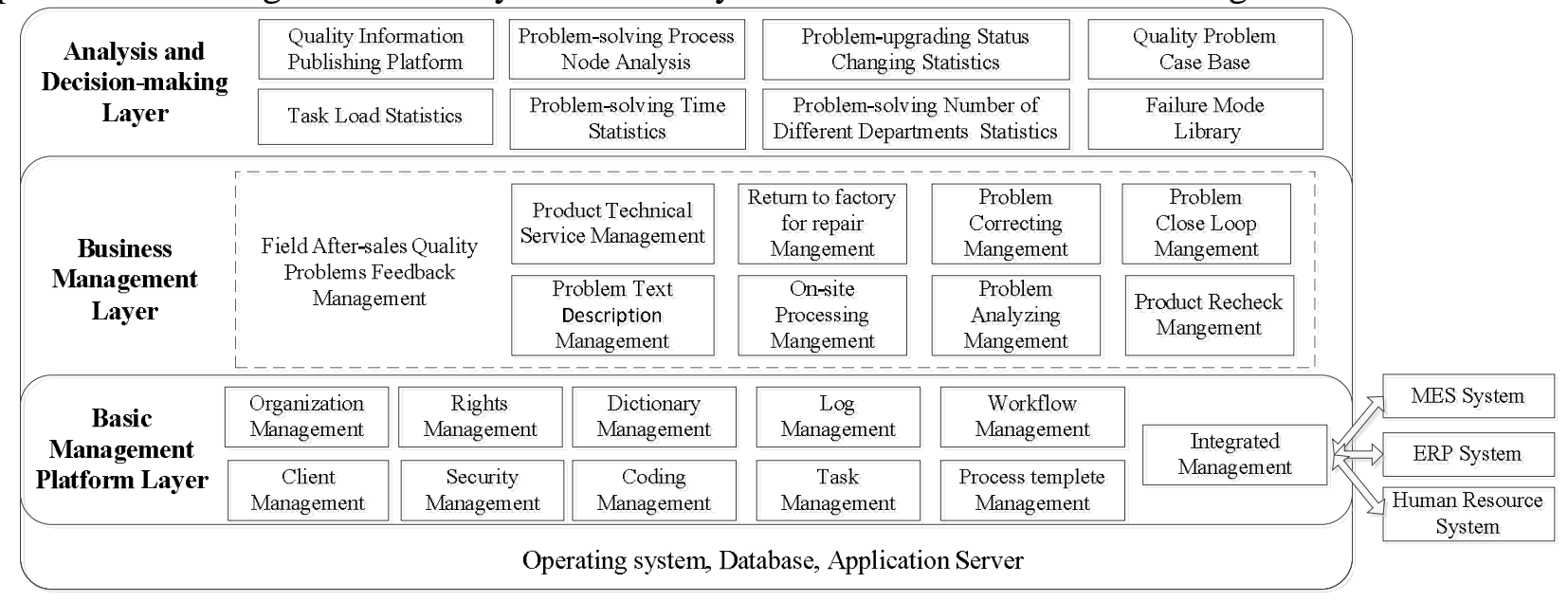

Fig.3 The multi-layer software system architecture

The system is a Web application system which is based on J2EE technology architecture, constructed by three layers: the basic platform layer, business management layer, analysis and decision-making layer.

Basic platform layer is the foundation of business management layer and analysis decision-making layer and its function includes organization management, user management, rights management, coding management, dictionary management, security management, log management, task management, workflow management, process template management and integration management and so on.

As for business management layer, its function contains field quality issues management, on-site service management, faulty product returned to factory for re-repair management, problem analysis management, problem corrective management, problem close loop management, problem learned by analogy management, product technical service management, problem description management.

As for analysis and decision-making layer, its functions not only include problem processing status changing management, the personal work load statistical analysis of different periods, but also some management decision support tools like quality problem case database, failure mode library which can facilitate the problem nature judgment of different levels and the choice of problem treatment.

In addition, the system can also be integrated with the MES system and ERP system so that the returned product information can be shared in time to ensure the consistency of the product information before and after the process and achieve the whole process management of the faulty product problem.

System Function Design. According to the above multi-layer software system architecture, the functions of different modules are briefly described as follows:

ü System management. Some basic system functions are only provided for system administrator, such as organization management, user management, rights management, security management, log management, task management, workflow management, process template management, integrated management. The system administrator can perform role location, rights assignment and some other 
system information management through these functions.

ü Basic information management. Basic information management, like coding management and dictionary management, are provided both for system administrators and data managers. The administrator can encode the module contents according to the needs of each service module. Meanwhile, the administrator can also use dictionary function to manage the commonly used words in order to facilitate the statistical analysis in later period.

ü Quality problems processing. The field quality problem feedback can be matched to different treatments according to the nature of the problem. With the help of Case Base and Failure mode library, the after-sales administrator can determine the nature of the problem and assign the appropriate treatment for the problem. For seriously affected quality problem processing, a variety of staffs from different departments will be involved in the collaborative problem-solving.

ü Case Base management for solved problems. After-sales quality administrators can use these two functions, Case Base and Failure mode library, to determine the problem level and choose available treatment for field quality problem. In addition, after the problem is solved, the typical problem will be added to the Case Base by after-sales administrator. And the different types of cases will be classified by different failure modes so that it is convenient for administrator to focus on the solution quickly and save time in adjusting problem treatments in later handlings.

ü Information Statistics and analysis. Some kinds of statistical analysis charts for different dimensions of field feedback problems are provided in this system, such as the statistic chart of the field feedback problem from different users by year, the statistic chart which shows the solved problem number of different departments during a certain time scale, etc. Meanwhile, advanced statistical analysis functions, such as node analysis of problem processing, time statistics of problem processing, etc., are also realized in this system.

\section{System Application and Evaluation}

A Brief Introduction of the Applied Enterprise. The aimed aerospace electrical connector manufacturing enterprise is the typical $R \& D$ electrical connector manufacturing enterprise. The enterprise produce various types of products, as a result, their after-sales quality problem management has these features: multi-sectoral collaboration, frequently changing quality problems treatment in processing mode, etc. The after-sales service business in this company has not yet adopted information technology, thus information collection and processing of different departments are mainly recorded in paper documents. Whether for historical information search, or information exchange between departments, there are only the traditional manual ways available for staffs to deal with information. This kind of situation cannot meet the refinement and quality improvement needs in after-sales problem management. Therefore, the enterprise needs to establish a set of product quality management system in order to achieve the high efficiency of after-sales quality problem processing, coordination and meticulous control, as well as the management process management decision support.

Application Effect Analysis. After-sales quality management information system has been put into use in the enterprise. Through the implementation of the system, the efficiency of the after-sales quality problem management has been greatly improved, and the original multi-processing business staggered management process has been optimized, and the refinement and quality improvement in after-sales problem processing management are achieved. The detailed management improvements in the applied enterprise can be seen in these six areas:

ü Case base knowledge accumulation: The after-sales administrator used Case Base to accumulate historical cases for the enterprise. For some typical cases, the administrator has classified them and transformed the important information into knowledge for further use, such as problem description, problem positioning, solutions, etc.;

ü Precise analysis of the problem location: The after-sales administrator can get help from the failure mode library and case base when receiving different problems. When the administrator typed 
keywords of the problem in failure mode library and case base, case retrieving can be accomplished rapidly and expediently. So the accuracy of the user needs analysis has been enhanced in this way;

ü Problem lifecycle management: For any quality problem, the administrator can view the problem lifecycle record to find various information of this problem, which helped the administrator to get a complete comprehension of the process for problem-solving;

ü Problem co-processing: For a complex problem which requires multi-departmental work, responsible departments received requests in time from other departments during the problem-solving process, and the quality management administrator checked different nodes' status in the workflow and give some recommendations to the relevant departments;

ü Traceability of the problem: For any quality problem, the administrator can use problem processing workflow to check different nodes in it. The administrator can trace the previous information step by step to find further information about this problem;

ü Quality management system improvements: Administrators can view charts and tables of different dimensions in the system, which helped the administrator to find out the bottleneck in current management.

\section{Conclusions}

This paper has studied the approach of after-sales quality problem management in order to improve the processing efficiency of after-sales quality problem for aerospace electrical connectors. The requirements of after-sales quality problem management under the information environment are analyzed. The process model of after-sales quality problem management is setup, in which the multi-types of quality problem handling method are considered. The multi-layer software system architecture for after-sales quality problem management is provided. The information management system of after-sales quality problem is developed by using J2EE architecture, and the feasibility and validity of the proposed method are verified by applied the software system in an aerospace electrical connector manufacturer. In the future, the author hopes to do further researches on the optimization methods of tasks delivery of after-sales quality problems and multivariate statistical analysis and evaluation methods for problem solving, so as to find a more optimal information solution for the enterprise to further improve efficiency of after-sales quality problem management.

\section{Acknowledgements}

This work was financially supported by the Quality Management Information System Project of Hangzhou Aerospace Electronic Technology Limited Corporation.

\section{References:}

[1] Chengbo Yang, Reliability Analysis of Aerospace Electrical Connector [J], China Plant Engineering, August 2006: 36-38, in Chinese.

[2] Sida Lin, An Introductory Review on Reliability Research of Electrical Connectors, Electromechanical Components, December 2009:52-56, in Chinese.

[3] Hau L. Lee, Ultimate Enterprise Value Creatioin Using Demand-Based Management, Stanford Global Supply Chain Management Forum, SGSCMF-W1-2001, September 2001.

[4] QJ 3057-98 Aerospace-Electrical, Electronic and Electromechanical (EEE) Components - Quality Assurance Requirements, in Chinese.

[5] Guideline of Quality Problem Close Loop Aerospace Product, Beijing (2003), in Chinese. 